\title{
Tratamento cirúrgico dos aneurismas de ventrículo esquerdo com reconstrução geométrica: aspectos cirúrgicos e resultados imediatos
}

\author{
Gilmar Geraldo dos SANTOS*, Victor L. S. HADDAD*, Silas F. AVELAR Jr., Antônio Amauri GROPPO, \\ Ricardo BEYRUTI*, Rosa Maria SIMŐES*, Noedir A. G. STOLF*
}

RBCCV $44205-140$

\begin{abstract}
SANTOS, G. G.; HADDAD, V. L. S.; AVELAR Jr., S. F.; GROPPO, A. A.; BEYRUTI, R.; SIMŌES, R. M.; STOLF, N. A. G. - Tratamento cirúrgico dos aneurismas de ventrículo esquerdo com reconstrução geométrica: aspectos cirúrgicos e resultados imediatos. Rev. Bras. Cir. Cardiovasc., 6(2): 116-123, 1991.

RESUMO: Os resultados imediatos de 79 pacientes operados num período de sete anos para tratamento cirúrgico de aneurisma de ventrículo esquerdo com a técnica de reconstrução geométrica circular são analisados. A indicaçāo cirúrgica mais freqüente foi a insuficiência cardíaca congestiva $(78,4 \%)$, isolada $(25,3 \%)$ ou associada à insuficiência coronária $(53,1 \%)$. Sessenta $(76 \%)$ pacientes estavam em classe funcional III e $10(12,6 \%)$ em classe funcional IV. Cinqüenta e oito $(73,4 \%)$ pacientes foram submetidos à revascularização do miocárdio concomitantemente. A mortalidade hospitalar foi de $5,1 \%$ e teve incidência maior nos pacientes com idade acima de $60(12 \%)$ anos, em classe funcional IV $(20 \%)$, com má função ventrícular (FE $<0,30-20 \%$ e PDFVE $>25-14 \%$ ) e lesōes coronárias triarteriais (10\%). Estes fatores também estiveram associados a complicaçōes cardiacas com baixo débito e uso de BIA. Outros fatores de risco citados na literatura são discutidos. Os resultados imediatos desse estudo e os resultados imediatos e tardios de outros em que a técnica usada foi semelhante são melhores que os obtidos com outros tipos de correção e sugerem ser este o procedimento de escolha para o tratamento dessa afeç̧ão.
\end{abstract}

DESCRITORES: aneurismas de ventrículo esquerdo, cirurgia, reconstrução geométrica.

\section{INTRODUÇĀO}

O aneurisma de ventrículo esquerdo é uma complicação na evolução do infarto do miocárdio transmural, que acomete $5-30 \%$ dos pacientes, sendo mais freqüente nas obstruçōes proximais da artéria descendente anterior ${ }^{1}$ 15, 32 .

O tratamento cirúrgico tem sido freqüentemente indicado com melhora da sintomatologia, qualidade de vida e sobrevida dos pacientes ${ }^{6,21,33}$. A insuficiência cardiaca congestiva, tromboembolismo arterial sistêmico e arrit- mias ventriculares são as principais indicações para a correçāo cirúrgica do aneurisma. Muitas vezes, no entanto, a correção do aneurisma é realizada concomitantemente com a revascularização do miocárdio, em pacientes anginosos.

Desde 1958, quando COOLEY et alii ${ }^{7}$ realizaram a primeira ressecção de aneurisma pós-infarto do ventrículo esquerdo usando circulação extracorpórea, várias técnicas de correção foram propostas, com resultados conflitantes e variáveis, e mortalidade elevada em alguns estudos.

\footnotetext{
Trabalho realizado na Unidade de Doenças Torácicas (Serviço Prof. Noedir Stolf). Hospital da Beneficência Portuguesa. São Paulo, SP, Brasil. Recebido para publicaçăo em 2 de maio de 1991.

- Da Unidade de Doenças Torácicas (Serviço Prof. Noedir Stolf). Hospital da Beneficência Portuguesa.

Endereço para separatas: Gilmar Geraldo dos Santos. Rua Maestro Cardim, 769. Sala 227. 01323 São Paulo, SP, Brasil.
} 
SANTOS, G. G.; HADDAD, V. L. S.; AVELAR Jr., S. F.; GROPPO, A. A.; BEYRUTI, R.; SIMŐES, R. M.; STOLF, N. A. G. Tratamento cirúrgico dos aneurismas de ventrículo esquerdo com reconstruçāo geométrica: aspectos cirúrgicos e resultados imediatos. Rev. Bras. Cir. Cardiovasc., 6(2): 116-123, 1991.

Esse estudo analisa a casuística e os resultados imediatos dos pacientes operados no Serviço do Prof. Dr. Noedir Stolf, no Hospital da Beneficência Portuguesa de Sāo Paulo, para tratamento cirúrgico do aneurisma do ventrículo esquerdo com a técnica geométrica proposta por JATENE ${ }^{18}$.

\section{CASUISTICA E MÉTODOS}

De outubro de 1983 a outubro de 1990,79 pacientes foram submetidos a aneurismectomia do ventrículo esquerdo com reconstrução geométrica isolada ou associada a revascularização do miocárdio. Sessenta e quatro $(81,0 \%)$ eram do sexo masculino, $77(97,4 \%)$ da raça branca, um $(1,3 \%)$ da raça negra e outro $(1,3 \%)$ da raça amarela. A idade média foi de 54,1 anos e variou de 32 a 74 anos. Ao exame clínico, $62(78,4 \%)$ pacientes apresentavam sintomas e sinais de insuficiência cardiaca congestiva como dispnéia aos pequenos e médios esforços ou em repouso com ou sem edema de membros inferiores. Destes, quatro $(5,1 \%)$ tiveram episódios de dispnéia paroxística noturna e três $(3,8 \%)$, edema agudo do pulmão. Vinte $(25,3 \%)$ pacientes apresentaram insuficiência cardiaca congestiva isolada e $42(53,1 \%)$, sintomas de insuficiência cardiaca congestiva e angina pectoris concomitante. Angina isolada foi encontrada em 16 $(20,3 \%)$ pacientes. Sessenta e dois $(78,4 \%)$ pacientes estavam medicados com digital, diuréticos ou vasodilatadores. Três $(3,8 \%)$ pacientes apresentavam síncope, um deles associada a arritmia ventricular grave. Arritmia foi observa em $13(16,5 \%)$ pacientes. Na época da operaçăo, nove $(11,4 \%)$ pacientes encontravam-se em classe funcional II, 60 (76\%) em classe funcional III e 10 (12,6\%) pacientes em classe funcional IV, segundo classificação da NYHA. Entre os pacientes em classe funcional II, seis tinham teste ergomético positivo.

$\mathrm{O}$ aneurisma do ventrículo esquerdo era pós-infarto do miocárdio em $78(98,7 \%)$ pacientes e em um $(1,3 \%)$ a etiologia era doença de Chagas. Setenta $(88,6 \%)$ pacientes haviam tido um episódio de infarto do miocárdio prévio, nove $(11,4 \%)$, dois ou mais infartos prévios. Sessenta e seis $(83,5 \%)$ pacientes tiveram infarto em região ântero-septal extenso, quatro $(5,1 \%)$ em região inferior, quatro $(5,1 \%)$ em regiōes anterior e lateral e cinco $(6,3 \%)$ em regiōes anterior $\mathrm{e}$ inferior.

O tempo médio entre o último episódio de infarto e a operação foi de 5,2 meses, variando de 28 dias a 36 meses.

A indicação cirúrgica foi devido a sintomas decorrentes da presença do aneurisma em 20 (25,3\%) pacientes (dispnéia, ICC, arritmia), por insuficiência coronária com angina pectoris em $16(20,3 \%)$, e por associação de ambas em $42(53,1 \%)$ pacientes. Em um (1,3\%) paciente a indicaçāo foi por arritmia ventricular grave e sintomática. Antecedentes de hipertensão arterial sistêmica foram encontrados em $40(50,6 \%)$ pacientes, de diabetes em $11(13,9 \%)$, neurológicos em três $(3,8 \%)$, doença de Chegas em dois $(2,6 \%)$, doença pulmonar obstrutiva crônica em quatro $(5,1 \%)$, insuficiência renal crônica em um $(1,3 \%)$ e isquemia em membros inferiores em um $(1,3 \%)$ paciente.

O eletrocardiograma mostrou alteraçōes com zona eletricamente inativa ântero-septal e supradesnivelamento do segmento ST isolados em $41(51,9 \%)$ pacientes, associada a alteraçōes isquêmicas em outras regiōes em $22(34,2 \%)$, zona inativa inferior em cinco $(6,3 \%)$, zona inativa anterior e inferior em cinco $(6,3 \%)$ e zona inativa anterior e lateral em dois $(2,6 \%)$ pacientes.

Ao exame radiográfico do tórax, a área cardíaca estava normal em $34(43,0 \%)$ pacientes, aumentada $+1++++$ em $26(32,9 \%)$, aumentada $++/++++$ $(22,8 \%)$ e $+++1++++$ em um $(1,3 \%)$ paciente. Sinais de congestão pulmonar foram observados em 27 $(34,2 \%)$ pacientes. O ecocardiograma foi realizado em $15(19 \%)$ pacientes e mostrou alterações na contratilidade do ventrículo esquerdo em todos os pacientes examinados, insuficiência mitral leve em quatro $(5,1 \%)$.

Todos os pacientes foram submetidos a estudo hemodinâmico, cinecoronariografia e cineventriculografia do ventrículo esquerdo. Vinte e um $(26,6 \%)$ pacientes tinham lesão em uma artéria, $27(34,2 \%)$ em duas artérias e $31(39,2 \%)$ em três ou mais artérias. A artéria descendente anterior (DA) estava ocluída em $66(83,5 \%)$ pacientes e recanalizada espontaneamente e associada a lesão em outra artéria em oito $(10,1 \%)$. Circulação colateral para a artéria DA de ++ ou $+++1++++$ foi encontrada em $13(16,5 \%)$ pacientes e de intensidade $+1++++$ em cinco $(6,3 \%)$ pacientes. Oclusão isolada da artéria DA estava presente em $21(26,6 \%)$ pacientes, associada a lesão severa em uma artéria em 15 (19\%), em duas artérias em $17(21,5 \%)$, em três artérias em um $(1,3 \%)$ e em tronco da coronária esquerda (TCE) em dois $(2,5 \%)$ pacientes. Oclusão combinada das artérias DA e circunflexa $(\mathrm{Cx})$ ou em seus ramos principais em cinco $(6,3 \%)$ pacientes e da artéria DA e artéria coronária direita (CD) em cinco $(6,3 \%)$ pacientes todos associados a lesão severa em uma terceira artéria. Oclusão da artéria CD estava presente em quatro $(5,1 \%)$ pacientes, associada a lesão em uma artéria em três $(3,8 \%)$, e a duas artérias em um (1,3\%) paciente. Lesão oclusiva total combinada das artérias CD e Cx foi encontrada em um $(1,3 \%)$ paciente associada a lesão na artéria DA.

Todos os pacientes apresentavam alterações na contratilidade do ventrículo esquerdo, sendo acinesia anterior em um $(1,3 \%)$, dilataçāo aneurismática e discinesia em parede anterior isolada em $65(82,3 \%)$, considerada moderada em $29(36,7 \%)$ e grande em $36(45,6 \%)$, associada a hipocinesia inferior ou lateral em oito $(10,1 \%)$. 
SANTOS, G. G.; HADDAD, V. L. S.; AVELAR Jr., S. F.; GROPPO, A. A.; BEYRUTI, R.; SIMŐES, R. M.; STOLF, N. A. G. Tratamento cirúrgico dos aneurismas de ventrículo esquerdo com reconstruçāo geométrica: aspectos cirúrgicos e resultados imediatos. Rev. Bras. Cir. Cardiovasc., 6(2): 116-123, 1991.

Discinesia em parede inferior isolada em quatro $(5,1 \%)$ e associada a acinesia lateral em um $(1,3 \%)$ paciente.

A funçāo do ventrículo esquerdo, avaliada pela fração de ejeção, estava levemente diminuída $(0.40<\mathrm{FE}$ $<0.50)$ em $44(55,7 \%)$ pacientes, moderadamente diminuída $(0.30<\mathrm{FE}<0.40)$ em $25(31,6 \%)$ e severamente diminuída ( $\mathrm{FE}<0,30)$ em $10(12,7 \%)$ pacientes. A pressão diastólica final do ventrículo esquerdo, medida em $\mathrm{mmHg}$, era de 10 a 20 em $31(39,2 \%)$ pacientes, de 21 a 25 em $26(32,9 \%)$, de 26 a 30 em $13(16,5 \%)$ e acima de 30 em nove $(11,4 \%)$ pacientes.

\section{Técnica Operatória}

Os pacientes foram operados através de toracotomia longitudinal e esternotomia mediana, circulação extracorpórea com cânula cavo-atrial única no átrio direito, canulaçāo da aorta ascendente e cateter de punção na aorta ascendente para infusão de solução cardioplégica e aspiração. Foi realizada hipotermia sistêmica moderada, sendo de $23^{\circ} \mathrm{C}$ nos pacientes submetidos a aneurismectomia isolada e a $28^{\circ} \mathrm{C}$ nos pacientes submetidos a revascularização do miocárdio concomitantemente; nestes, foi feita também hipotermia tópica com soro fisiológico gelado. A operação foi realizada através de pinçamento intermitente da aorta nos pacientes com aneurismectonia isolada e combinada com cardioplegia gelada infundida na raiz da aorta nos demais pacientes.

As operaçōes foram conduzidas segundo a técnica descrita por JATENE ${ }^{18}$, com pequenas modificaçōes. Após início da circulação extracorpórea os pacientes eram mantidos em hipotermia a $32^{\circ} \mathrm{C}$, com batimentos cardíacos preservados e pinçamento intermitente da aorta ascendente. A região aneurismática era identificada e a ventriculotomia esquerda, feita sobre a área de fibrose. Ainda com batimentos cardiacos presentes a região de fibrose era delimitada por palpação digital. Realizado, então, o pregueamento da região fibrosada do septo interventricular e, em seguida, cerclagem do colo do aneurisma com sutura em bolsa na regiāo de transição entre a fibrose e a região contrátil. Eram passados pontos em $U$ separados de Ethicon $O$ apoiados em tiras de Teflon na região delimitada pela sutura em bolsa fechando-se a ventriculotomia diretamente, sem uso de remendo. O excesso de músculo fibrosado era ressecado e a sutura reforçada com pontos em $X$, usando-se os mesmos fios passados anteriormente. $\mathrm{O}$ ar era retirado através de aspiração na aorta ascendente.

Os pacientes submetidos a revascularização do miocárdio concomitantemente eram resfriados até $28^{\circ} \mathrm{C}$ após a correção do aneurisma, a aorta ascendente pinçada e a soluçāo cardioplégica gelada (St. Thomas I) a $4^{\circ} \mathrm{C}$ infundida na raiz da aorta, sendo repetida a cada 20 minutos. As anastomoses distais, mamária-coronária ou safena-coronária eram realizadas.
Após as anastomoses distais, os enxertos venosos eram perfundidos através de derivação da linha arterial da circulação extracorpórea, a aorta despinçada e o paciente reaquecido. As anastomoses proximais safenaaorta eram realizadas com pinçamento intermitente ou lateral da aorta ascendente. Em oito pacientes a artéria DA foi revascularizada e irrigada antes da delimitação do aneurisma, reduzindo a massa muscular a ser ressecada. A pressão do átrio esquerdo foi monitorizada em todos os pacientes e o cateter de Swan-Ganz foi usado quando havia baixo débito cardíaco.

Em $21(26,6 \%)$ pacientes foi realizada somente a correção do aneurisma do ventrículo esquerdo e em 58 $(73,4 \%)$ foi realizada correção do aneurisma e revascularizaçāo do miocárdio concomitante. Foi usado enxerto de artéria mamária isolada em dois $(2,5 \%)$ pacientes, artéria mamária e veia safena autógena em oito $(10,1 \%)$ e apenas veia safena em $48(60,8 \%)$. Vinte e sete $(34,2 \%)$ pacientes receberam uma ponte, $21(26,6 \%)$ receberam duas pontes e $10(12,7 \%)$ pacientes receberam três pontes. A revascularizaçăo foi incompleta em oito $(10,15)$ pacientes com lesões em ramos secundários e de pequeno calibre.

As artérias que irrigavam a região do aneurisma foram revascularizadas sempre que a área englobada na correção era menor e se mantinham pérvias. Vinte e quatro $(30,4 \%)$ pacientes receberam enxerto na região do aneurisma, todos em parede anterior, quer na artéria DA ou em seus ramos diagonais.

$\mathrm{Na}$ correçāo isolada do aneurisma de ventrículo esquerdo o tempo de circulação extracorpórea (CEC) foi de até 30 minutos em cinco $(6,3 \%)$ pacientes, de 30 a 60 minutos em $14(17,7 \%)$, e entre 60 e 90 minutos em dois $(2,5 \%)$ pacientes. Nestes pacientes, o tempo de pinçamento da aorta foi igual ou menor que 10 minutos em $18(22,8 \%)$ e entre 10 e 30 minutos em três $(3,8 \%)$ pacientes.

Nos pacientes submetidos a correçāo do aneurisma e revascularização do miocárdio com uma ponte, o tempo médio de CEC foi de 75 minutos e de pinçamento da aorta de 30 minutos, com duas pontes, de 90 e 60 minutos e com três pontes, de 120 e 90 minutos, respectivamente. Todos os pacientes que apresentaram complicaçōes intra-operatórias (baixo débito cardiaco, arritmias) tiveram tempo de CEC superior a 120 minutos.

\section{RESULTADOS}

As características demográficas dos pacientes sāo semelhantes às de outros estudos, com maior incidência para o sexo masculino e idade média acima de 50 anos ${ }^{3}$. $A$ idade avançada esteve relacionada a maior índice de mortalidade (Tabela 1). Quanto ao quadro clínico e à 
SANTOS, G. G.; HADDAD, V. L. S.; AVELAR Jr., S. F.; GROPPO, A. A.; BEYRUTI, R.; SIMŌES, R. M.; STOLF, N. A. G. Tratamento cirúrgico dos aneurismas de ventrículo esquerdo com reconstrução geométrica: aspectos cirúrgicos e resultados imediatos. Rev. Bras. Cir. Cardiovasc., 6(2): 116-123, 1991.

TABELA 1

MORTALIDADE HOSPITALAR E COMPLICAÇÖES CARDIACAS RELACIONADAS A IDADE

\begin{tabular}{|c|c|c|c|c|c|}
\hline \multirow[t]{2}{*}{ Idade } & \multirow[b]{2}{*}{$N^{\circ}$} & \multicolumn{2}{|c|}{ Mortalidade } & \multicolumn{2}{|c|}{ Complicaçōes } \\
\hline & & $N^{\circ}$ & $\%$ & $N \cdot$ & $\%$ \\
\hline $30-39$ & 5 & 0 & 0 & 0 & 0 \\
\hline $40-49$ & 21 & 1 & $4,8 \%$ & 1 & $4,8 \%$ \\
\hline $50-59$ & 28 & 0 & 0 & 2 & $7,1 \%$ \\
\hline $60-69$ & 20 & 2 & $10,0 \%$ & 2 & $10,0 \%$ \\
\hline $70-79$ & 5 & 1 & $20,0 \%$ & 0 & 0 \\
\hline Total & 79 & 4 & $5,1 \%$ & 5 & $6,3 \%$ \\
\hline
\end{tabular}

TABELA 2

MORTALIDADE HOSPITALAR E COMPLICAÇÖES CARDIACAS RELACIONADAS AOS SINTOMAS PRÉ-OPERATORIOS

\begin{tabular}{|c|c|c|c|c|c|}
\hline \multirow[t]{2}{*}{ Sintomas } & \multirow[b]{2}{*}{$N^{0}$} & \multicolumn{2}{|c|}{ Mortalidade } & \multicolumn{2}{|c|}{ Complicaçōes } \\
\hline & & $N^{\circ}$ & $\%$ & $N^{\circ}$ & $\%$ \\
\hline - Angina isolada & 17 & 0 & 0 & 0 & 0 \\
\hline- ICC & 20 & 1 & $5,0 \%$ & 2 & $10,0 \%$ \\
\hline - ICC e angina & 42 & 3 & $7,1 \%$ & 3 & $7,1 \%$ \\
\hline - Arritmia ventric. & 1 & 0 & & 0 & 0 \\
\hline Total & 79 & 4 & $5,1 \%$ & 5 & $6,3 \%$ \\
\hline
\end{tabular}

TABELA 3

MORTALIDADE HOSPITALAR E COMPLICACOẼES CARDIACAS RELACIONADASA CLASSE FUNCIONAL (NYHA)

\begin{tabular}{|c|c|c|c|c|c|}
\hline \multirow[t]{2}{*}{ CF NYHA } & \multirow[b]{2}{*}{$N_{0}$} & \multicolumn{2}{|c|}{ Mortalidade } & \multicolumn{2}{|c|}{ Complicaçōes } \\
\hline & & $N^{\circ}$ & $\%$ & N: & $\%$ \\
\hline 1 & 0 & 0 & 0 & 0 & 0 \\
\hline II & 9 & 0 & 0 & 0 & 0 \\
\hline III & 60 & 2 & $2,3 \%$ & 3 & $5,0 \%$ \\
\hline IV & 10 & 2 & $20,0 \%$ & 2 & $20,0 \%$ \\
\hline 79 & 4 & $5,1 \%$ & 5 & $6,3 \%$ & \\
\hline
\end{tabular}

indicação de cirurgia, a insuficiência cardiaca congestiva e angina pectoris predominaram e a maioria dos pacientes estava em classe funcional III (Tabelas 2 e 3 ).

A parede anterior $e$ as artérias que a irrigam estiveram acometidas em grande parte dos pacientes $(93,7 \%)$.

Nenhum paciente faleceu na sala de operaçōes ou nas primeiras $\mathbf{4 8}$ horas de pós-operatório. A mortalidade hospitalar foi de $5,1 \%$, (quadro óbitos). Dois $(50 \%)$ pacientes tiveram óbito relacionado a causas cardíacas, baixo débito cardíaco importante, uso de balão intra- aórtico e drogas vasoativas. Destes, um paciente faleceu no 5 : dia de pós-operatório por falência ventricular e outro no 15 : dia de pós-operatório por insuficiência renal aguda e insuficiência respiratória. Um $(25 \%)$ paciente teve baixo débito cardiaco moderado, controlado apenas com drogas, apresentou distúrbios neurológicos e pulmonares e faleceu no 25 . dia de pós-operatório por sangramento digestivo maciço e um $(25 \%)$ paciente apresentou quadro de insuficiência respiratória e insuficiência renal aguda não relacionada a baixo débito cardíaco. Três desses pacientes tinham idade superior a 65 anos e todos estavam em classe funcional III ou IV com fração de ejeção severamente diminuída em dois e moderadamente diminuída em dois. Todos receberam revascularização do miocárdio completa associada.

Complicaçōes importantes no período de pós-operatório imediato foram verificadas em cinco pacientes. Três $(3,8 \%)$ pacientes tiveram síndrome de baixo débito cardíaco importante necessitando de balão intra-aórtico e catecolaminas em doses elevadas e dois $(2,5 \%)$ evoluíram com baixo débito cardíaco moderado necessitando apenas de catecolaminas. Vinte $(25,3 \%)$ receberam dopamina em dose baixa associada a vasodilatador (nitroprussiato de sódio), porém sem caracterizar quadro de débito cardíaco diminuído. Outras complicaçōes verificadas em $26(32,9 \%)$ pacientes foram arritmia ventricular ou atrial em $11(13,9 \%)$ pacientes, insuficiência respiratória em dois $(2,5 \%)$, broncopneumonia em dois $(2,5 \%)$, derrame pleural em um $(1,3 \%)$, insuficiência renal aguda em dois $(2,5 \%)$, insuficiência renal crônica agudizada e acidente vascular cerebral em um $(1,3 \%)$, complicaçōes neurológicas com confusão mental ou acidente vascular pulmonar em cinco $(6,3 \%)$ e sangramento necessitando reoperação para revisão de hemostasia em dois pacientes $(2,5 \%)$ e insuficiência cardiaca congestiva em quatro $(5,1 \%)$.

O período de internação hospitalar pós-operatória foi menor que dez dias em $44(58,7 \%)$ pacientes, entre dez e 15 dias em $25(33,3 \%)$ pacientes, entre 15 e 20 dias em dois pacientes e entre 21 e 28 dias em quatro $(5,3 \%)$ pacientes.

Nenhum paciente em classe funcional II, fração de ejeçāo normal ou levemente diminuída (FE $>0.40)$ e pressāo diastólica final do ventrículo esquerdo de até $200 \mathrm{mmHg}$ teve complicação relacionada ao débito cardíaco no pós-operatório imediato. Entre os pacientes com insuficiência cardíaca e ou angina em classe funcional III $(60-76 \%)$, cinco $(8,3 \%)$ apresentaram síndrome de baixo débito cardiaco importante no período de pós-operatório imediato e três necessitaram balão intra-aórtico, todos com fração de ejeção moderada ou severamente diminuída e pressão diastólica final de ventrículo esquerdo superior a $25 \mathrm{mmHg}$. Dos pacientes em classe funcional III, dois $(3,3 \%)$ faleceram, em um deles a evolução não esteve relacionada a baixo débito cardiaco. En- 
SANTOS, G. G.; HADDAD, V. L. S.; AVELAR Jr., S. F.; GROPPO, A. A.; BEYRUTI, R.; SIMÓES, R. M.; STOLF, N. A. G. Tratamento cirúrgico dos aneurismas de ventrículo esquerdo com reconstrução geométrica: aspectos cirúrgicos e resultados imediatos. Rev. Bras. Cir. Cardiovasc., 6(2): 116-123, 1991.

tre os pacientes em classe funcional IV $(10 \%-12,6 \%)$, três $(30 \%)$ tiveram baixo débito cardiaco no período de pós-operatório e necessitaram balão intraaórtico, todos com fração de ejeção menor ou igual a 0.30 e pressão diastólica final do ventrículo esquerdo acima de 28 $\mathrm{mmHg}$. Destes, dois (20\%) pacientes faleceram (Tabelas 3 e 4).

O balāo intraaórtico foi usado em cinco $(6,3 \%)$ pacientes e o tempo de manutenção variou de 24 a 72 horas e nenhum paciente teve complicaçōes relacionadas ao seu uso; dois ( $40 \%$ ) pacientes faleceram.

Nenhum paciente submetido a aneurismectomia isolada do ventrículo esquerdo faleceu $(21-26,6 \%)$ $(0 \%)$, três $(14,3 \%)$ pacientes tiveram ramos arteriais secundários e finos com lesão moderada nāo revascularizados. Seis $(28,6 \%)$ pacientes tinham a função do ventrículo esquerdo normal ( $F E>0.50)$ e a pressão diastólica final do ventrículo esquerdo (Pdf VE) menor que $20 \mathrm{mmHg}$, quatro (19\%), tinham a função do VE levemente diminuída $(0.40<\mathrm{FE}<0.50)$ e a Pdf VE entre 20 e $25 \mathrm{mmHg}$, seis $(28,6 \%)$ tinham a função de VE moderadamente diminuída $(0.30<\mathrm{FE}<0.40)$ e dois $(9,5 \%)$ tinham a funçāo de VE severamente diminuída (FE $<0.30$ ), todos com Pdf VE entre 25 e $35 \mathrm{mmHg}$.

TABELA 4

MORTALIDADE HOSPITALAR E COMPLICAÇÓES CARDIACAS RELACIONADASA FUNCYÃO DO VENTRICCULO ESQUERDO

\begin{tabular}{|c|c|c|c|c|c|}
\hline \multirow[t]{2}{*}{ Variáveis } & \multirow[b]{2}{*}{$N \cdot$} & \multicolumn{2}{|c|}{ Mortalidade } & \multicolumn{2}{|c|}{ Complicaçōes } \\
\hline & & $N^{\circ}$ & $\%$ & No & $\%$ \\
\hline $\mathrm{FE}>0.40$ & 44 & 0 & 0 & 0 & 0 \\
\hline $0.30<\mathrm{FE}<0.40$ & 25 & 2 & $8,0 \%$ & 3 & $12,0 \%$ \\
\hline $\mathrm{FE}<0.30$ & 10 & 2 & $20,0 \%$ & 2 & $20,0 \%$ \\
\hline PDFVE $<20$ & 31 & 0 & 0 & 1 & $3,1 \%$ \\
\hline $20<$ PDfVE $<25$ & 26 & 1 & $3,8 \%$ & 0 & 0 \\
\hline $25-$ PDfVE $<30$ & 13 & 3 & $23,0 \%$ & 3 & $23,0 \%$ \\
\hline PDfVE $>30$ & 9 & 0 & 0 & 1 & $11,1 \%$ \\
\hline
\end{tabular}

- FE; fração de ejeção (em \%)

- PVfDE: pressão diastólica final de ventrículo esquerdo em $\mathrm{mmHg}$ )
Dos pacientes submetidos a aneurismectomia e revascularizaçāo do miocárdio com uma ponte (27 $34,2 \%)$, quatro $(14,8 \%)$ apresentam síndrome de baixo débito cardíaco no período de pós-operatório e dois $(7,4 \%)$ necessitaram uso de balão intraórtico. Destes pacientes, três apresentavam função de VE moderadamente diminuida $(0.30<\mathrm{FE}<0.40)$ e um severamente diminuida ( $\mathrm{FE}<0.30$ ) e Pdf VE entre 25 e $35 \mathrm{mmHg}$. Neste grupo, um $(3,7 \%)$ paciente faleceu no 25 : dia de pós-operatório. Entre os pacientes com aneurismectomia e revascularização com duas pontes $(21-26,6 \%)$, três $(14,3 \%)$ tiveram baixo débito cardiaco importante e um $(4,8 \%)$ teve baixo débito cardíaco moderado, dois $(9,5 \%)$ pacientes necessitaram balão intraaórtico. Destes pacientes, dois tinham a função do VE moderadamente diminuída $(0.30<\mathrm{FE}<0.40)$ e Pdf VE entre 20 e 25 $\mathrm{mmHg}$ e dois pacientes com função de VE severamente diminuída ( $F E<0.30$ ) e Pdf VE entre 25 e $35 \mathrm{mmHg}$. Dois $(9,5 \%)$ pacientes faleceram no período de pós-operatório. Entre os pacientes com aneurismectomia e três pontes $(10-12,7 \%)$, um $(10 \%)$ apresentou baixo débito cardíaco importante, sem uso de balão intra-aórtico. Apresentava função de VE severamente diminuída (FE $=0.30)$ e Pdf VE $=28 \mathrm{mmHg}$. Este paciente faleceu no 14: dia do pós-operatório por sangramento digestivo importante. Os pacientes que apresentaram complicaçōes intra-operatórias (baixo débito) tiveram tempo de circulação extracorpórea maior (>120 minutos), o que certamente contribuiu para a evolução desfavorável.

\section{COMENTÁRIOS}

As técnicas insavias no manuseio do infarto agudo do miocárdio (terapia trombolítica, angioplastia coronária e cirurgia) podem evitar ou reduzir a extensão da lesão miocárdica e a formação do aneurisma do ventrículo esquerdo. Porém, quando o paciente apresenta esta complicação e se mantém sintomático, o tratamento cirúrgico apresenta resultados melhores que o tratamento clínico, quanto ao alívio dos sintomas, melhora da qualidade de vida e sobrevida ${ }^{3}, 11,13,14,19,21,30,33$.

TABELA 5

MORTALIDADE HOSPITALAR E COMPLICAÇÓES CARDIACAS RELACIONADAS AOS PROCEDIMENTOS EFETUADOS

\begin{tabular}{|c|c|c|c|c|c|c|c|}
\hline \multirow[t]{2}{*}{ Procedimento } & \multirow[t]{2}{*}{$N:$} & \multicolumn{2}{|c|}{ Mortalidade } & \multirow[t]{2}{*}{$F E$} & \multicolumn{2}{|c|}{ Complicaçōes } & \multirow[t]{2}{*}{$F E$} \\
\hline & & $N^{0}$ & $\%$ & & $N^{0}$ & $\%$ & \\
\hline Aneurismectomia VE isolada & 21 & 0 & 0 & & 0 & 0 & \\
\hline Aneurismectomia VE e 1 ponte & 27 & 1 & $3,7 \%$ & $(0.35)$ & 2 & $7,4 \%$ & (0.39) \\
\hline Aneurismectomia VE e 2 pontes & 21 & 2 & $9,5 \%$ & $(0.38$ e 0.30$)$ & 3 & $14,3 \%$ & $(<0.30(2)$ e $0.35(1))$ \\
\hline Aneurismectomia VE e 3 pontes & 10 & 1 & $10,0 \%$ & $(0.30)$ & 0 & 0 & \\
\hline
\end{tabular}


SANTOS, G. G.; HADDAD, V. L. S.; AVELAR Jr., S. F.; GROPPO, A. A.; BEYRUTI, R.; SIMÕES, R. M.; STOLF, N. A. G. Tratamento cirúrgico dos aneurismas de ventrículo esquerdo com reconstrução geométrica: aspectos cirúrgicos e resultados imediatos. Rev. Bras. Cir. Cardiovasc., 6(2): 116-123, 1991.

A demora no diagnóstico e na intervenção cirúrgica reduz as chances de sobrevida e aumenta os riscos de complicaçōes tardias ${ }^{21}$.

Desde os primórdios da cirurgia cardiaca e até os dias atuais o tratamento cirúrgico do aneurisma do ventrículo esquerdo tem apresentado mortalidade elevada na maioria das casuísticas encontradas na literatura e varia de $2 \%$ a $19 \%$ (média de $9,9 \%$ ) $2,4,5,8-10,16,18-20,23-25$. 27, 28, 31.

Muitos fatores de risco foram identificados e associados à mortalidade elevada, porém a técnica usada na correção do aneurisma é certamente um fator importante no resultado cirúrgico imediato, ao lado do estado pré-operatório do paciente.

O sucesso da correção depende de boa função cardíaca no período de pós-operatório e está associada à reconstrução correta da cavidade ventricular esquer$\mathrm{da}^{12,17}$. Neste sentido, a técnica descrita por JATENE ${ }^{18}$ e utilizada nessa série de pacientes é adequada por permitir avaliar com precisão a extensão da lesão muscular e a massa muscular a ser ressecada, corrige as distensōes e discinesias septais, evita suturas longas e lineares que deformam o coração e elimina, na maior parte dos casos, o uso do remendo para o fechamento da ventriculotomia. Pouca diferença hemodinâmica foi verificada entre a correção linear longa e o uso do remendo em estudo experimental por NICOLOSI et alii ${ }^{26}$, ambos eliminados com a técnica usada.

A mortalidade hospitalar global da presente série foi de $5,1 \%$, abaixo da média encontrada na literatura $(9,9 \%)$ e semelhante aos primeiros resultados apresentados com esta técnica $(4,3 \%)^{18}$ e superior aos resultados de outras técnicas propostas ${ }^{7}, 12,32$. Não houve mortalidade hospitalar para o tratamento do aneurisma de ventrículo esquerdo isolado $(0 \%)$ e para o tratamento do aneurisma do ventrículo esquerdo associado à revascularização do miocárdio a mortalidade hospitalar foi de $6,8 \%$ contra $2,3 \%$ e $5,0 \%$ respectivamente, relatados na literatura. As causas cardíacas estiveram relacionadas, direta ou indiretamente, a $75 \%$ dos óbitos, e o baixo débito cardíaco por falência aguda do miocárdio foi a principal causa, fato observado por outros autores ${ }^{9,32}$ 34 .
Os fatores de risco identificados foram a extensão da doença coronária com lesão em três ou mais artérias, a extensão do infarto prévio refletido no estado clínico do paciente com classe funcional IV, presença de sintomas de insuficiência cardiaca congestiva importante com ou sem angina, função miocádica severamente diminuida (FE $<0.30$ e Pdf VE $>25$ ) e idade acima de 65 anos. Estes fatores de risco, isolados ou associados, estavam presentes em todos os pacientes falecidos e são citados em vários artigos publicados ${ }^{3.14,34}$.

O número de pacientes que apresentou baixo débito cardíaco importante no período de pós-operatório e necessitou uso de balão intraaórtico $(6,3 \%)$ ou drogas vasoativas por tempo prolongado $(2,5 \%)$, foi menor que o encontrado em outros estudos sobre o apoio mecânico com balão intraaórtico ou outros aparelhos foi usado em $7 \%$ a $50 \%$ dos pacientes ${ }^{9,12,32,34}$.

Entre outros fatores de risco apontados freqüentemente, a taquiarritmia e a arritmia ventricular ${ }^{4,29}$ estiveram presentes em apenas um paciente operado que sobreviveu com melhora acentuada da sintomatologia. Nenhum paciente foi operado antes de $\mathbf{2 8}$ dias do último episódio de infarto agudo do miocárdio ${ }^{25}$, nenhum paciente foi operado em caráter de emergência ${ }^{9}$ e oito $(10,1 \%)$ receberam revascularização incompleta, excluindo-se a região aneurismática ${ }^{25}$. Deve-se ressaltar que $24(30,4 \%)$ dos pacientes receberam enxertos na regiẫo do aneurisma, o que diminui a extensão da ressecção e melhora a contratilidade das áreas adjacentes. O remendo de Dacron ou pericárdio bovino não foi usado nestes pacientes e esteve relacionado à maior mortalidade em outros trabalhos ${ }^{12,18}$.

O estudo completo e o preparo pré-operatório adequado dos pacientes, o esforço para afastar as causas que aumentam o risco operatório sempre que possível e a condução cuidadosa do período de pós-operatório contribuíram para os resultados obtidos.

Os resultados imediatos observados no presente estudo sugerem que a técnica de correção do aneurisma do ventrículo esquerdo com reconstrução geométrica é o procedimento de escolha para o tratamento da afecção. 
SANTOS, G. G.; HADDAD, V. L. S.; AVELAR Jr., S. F.; GROPPO, A. A.; BEYRUTI, R.; SIMŌES, R. M.; STOLF, N. A. G. Tratamento cirúrgico dos aneurismas de ventrículo esquerdo com reconstrução geométrica: aspectos cirúrgicos e resultados imediatos. Rev. Bras. Cir. Cardiovasc., 6(2): 116-123, 1991.

RBCCV 44205-140

SANTOS, G. G.; HADDAD, V. L. S.; AVELAR Jr., S. F.; GROPPO, A. A.; BEYRUTI, R.; SIMÓES, R. M.; STOLF, N. A. G. - Left ventricular aneurysmectomy with geometric reconstruction: surgical aspects and immediate results. Rev. Bras. Cir. Cardiovasc., 6(2): 116-123, 1991.

ABSTRACT: The early results of 79 patients who underwent left ventricular aneurysmectomy with the geometric and circular reconstruction in a seven years experience are analyzed. The most common indication for operation was congestive heart failure $(\mathbf{7 8 . 4} \%)$, isolated $(25.3 \%)$ or with coronary artery disease $(53.1 \%)$. Sixty $(76 \%)$ patients were in NYHA class III and $10(12.6 \%)$ in NYHA class IV at the time of surgery. Fifty eight $(73.4 \%)$ underwent coronary artery bypass graft surgery. Hospital mortality was $5.1 \%$ and patients older than $60(12 \%)$, in NYHA class IV $(20 \%)$, with poor left ventricular function (FE $<0.30-20 \%$ and LVEDP $>25-14 \%$ ) and extensive coronary artery disease $(10 \%)$ were under increased risk. Low cardiac output and use of IABP were also associate with this risk factors. Other factors of increased risk pointed in the literature are discussed. There were no deaths for isolated left ventricular aneurysmectomy. The early results of this study and the early and late results from others using the same technique are better than that obtained by others with different types of correction and they suggest that this is the procedure of choice to treat left ventricular aneurysm.

DESCRIPTORS: aneurysms of left ventricle, surgery, geometric reconstruction.

\section{REFERÊNCIAS BIBLIOGRÁFICAS}

1 ABRAMS, D. L.; EDELIST, A.; LURIA, M. H.; MILLER, A. J. - Ventricular aneurysm: a reappraisal based on a study of sixty-five consecutive autopsied cases. Circulation, 27: 164-169, 1963.

2 AKINS, C. W. - Resection of left ventricular aneurysm during hipotermic fibrillatory arrest without aortic occlusion. J. Thorac. Cardiovasc. Surg., 91: 610-618, 1986.

3 BARRATT-BOYES, B. G.; WHITE, H. D.; AGNEW, T. M.; PEMBERTON, J. R.; WILD, C. J. - The results of surgical treatment of left ventricular aneurysms: an assessment of the risk factors affecting early and late mortality. J. Thorac. Cardiovasc. Surg., 87: 87-98, 1984.

4 BRAWLEY, R. K.; MAGOVERN, C. J.; GOTT, V. L.; DONAHOO, J. S.; GARDNER, T. J.; WATKINS, L. - Left ventricular aneurysmectomy: factors influencing postoperative results. J. Thorac. Cardiovasc. Surg., 85: 712-717, 1983.

5 BURTON, N. A.; STINSON, E. B.; OYER, P. E.; SHUMWAY, N. E. - Left ventricular aneurysm: preoperative risk factors and long-term postoperative results. J. Thorac. Cardiovasc. Surg., 77: 65-75, 1979.

6 COHEN, M.; PACKER, M.; GOLIN, R. - Indications for left ventricular aneurysmectomy. Circulation, 67: 712-722, 1983.

7 COOLEY, D. A.; COLLINS, H. A.; MORRIS, G. C. - Left ventricular aneurysm after myocardial infarction: surgical excision with use of temporary cardiopulmonary bypass. JAMA, 167: 557-580, 1958.
8 GOSGROVE, D. M.; LOOP, E. D.; IRARRAZAVAL, M. J.; GROVES, L. K.; TAYLOR, P. C.; GOLDING, L. A. Determinants of longterm survival after ventricular aneurysmectomy. Ann. Thorac. Surg., 26: 357-363, 1978.

9 COSGROVE, D. M.; LYTLE, B. W.; TAYLOR, P. C.; STEWART, R. W.; GOLDING, L. A. R.; MAHFOOD, S.; GOORMASTIC, M.; LOOP, F. D. - Ventricular aneurysm resection: trends in surgical risk. Circulation, 79 (Supl. 1): 97-101, 1989.

10 CROSBY, I. K.; WELLONS, H. A.; MARTIN, R. P.; SCHUCH, D.; MULLER, W. H. - Employability: a new indication for aneurysmectomy and coronary revascularization. Circulation, 62 (Supl. 1): 79-83, 1980.

11 DAVIS, R. W. \& EBERT, P. A. - Ventricular aneurysm: a clinical-pathologic correlation. Am. J. Cardiol., 29: 1-6, 1972.

12 DOR, V.; SAAB, M.; COSTE, P.; KORNASZEWSKA, M.; MONTIGLIO, F. - Left ventricular aneurysm: a new surgical approach. Thorac. Cardiovasc. Surg., 37: $11-19,1989$.

13 FAXON, D. P.; RYAN, T. J.; DAVIS, K. B.; MCCABE, C. H.; MEYRS, W.; LESPERANCE, J.; SHAW, R.; TONG, T. G. - Prognostic significance of angiographically documented left ventricular aneurysm from the coronary artery surgery study (CASS). Am. J. Cardiol., 50: 157-164, 1982.

14 GONZALES-SANTOS, J. M.; ENNABLI, K.; GALINANES, X.; BOSH, J.; LESPERANCE, J.; PELLETIER, L. C. - Surgical treatment of the post-infarction left ventricular aneurysm: factors influencing early and late results. Thorac. Cardiovasc. Surg., 33: 86-93, 1985. 
SANTOS, G. G.; HADDAD, V. L. S.; AVELAR Jr., S. F.; GROPPO, A. A.; BEYRUTI, R.; SIMŌES, R. M.; STOLF, N. A. G. Tratamento cirúrgico dos aneurismas de ventrículo esquerdo com reconstrução geométrica: aspectos cirúrgicos e resultados imediatos. Rev. Bras. Cir. Cardiovasc., 6(2): 116-123, 1991.

15 GORLIN, R.; KLIEN, M. D.; SULLIVAN, J. M. - Mechanistic concept and clinical recognition. Am. J. Med. 42: 512-523, 1967.

16 HARKEN, A. H.; HOROWTZ, L. N.; JOSEPHSON, M. E. - Comparison of standard aneurysmectomy with directed endocardial resection for the treatment of recurrent sustained ventricular tachycardia. J. Thorac. Cardiovasc. Surg., 80: 527-534, 1980.

17 HUTCHING, G. M. \& BRAWLEY, R. D. - The influence of cardiac geometry on the result of ventricular aneurysm repair. Am. J. Pathol., 99: 221-228, 1980.

18 JATENE, A. D. - Left ventricular aneurysmectomy: ressection or reconstruction. J. Thorac. Cardiovasc. Surg., 89: 321-331, 1985.

19 JONES, E. L.; CRAVER, J. M.; HURST, J. W.; BRADFORD, J. A.; BONE, D. K.; ROBINSON, P. H.; COBBS, B. W.; THOMPKINS, T. R.; HATCHER Jr., C. R. Influence of left ventricular aneurysm on survival following the coronary artery bypass operation. Ann. Surg., 193: 733-742, 1981.

20 LEFEMINE, A. A.; GROVINDARAJAN, R.; RAMASWAMY, D.; BLACK, H.; MALDOFF, I.; SANELLA, N. - Left ventricular ressection for aneurysm and akinesia due to coronary artery disease: fifty consecutive patients. Ann. Thorac. Surg., 23: 461-466, 1977.

21 LOUAGIE, Y.; ALOUINI, T.; LESPERANCE, J.; PELLETIER, L. C. - Left ventricular aneurysm with predominanting congestive heart failure: a comparative study of medical and surgical treatment. J. Thorac. Cardiovasc. Surg., 94: 571-581, 1987.

22 MAGOVERN, G. J.; SAKERT, T.; LAUB, G. W.; LIEBLER, G.; BURKHOLDER, J.; BENCKART, D.; MAGOVERN Jr., G. J. - Surgical therapy for left ventricular aneurysms: a ten year experience. Circulation, 79 (Supl. 1): 102-107, 1989.

23 MORAN, J. M.; SCANLON, P. J.; NEMICKAS, R.; PIFAR RÉ, R. - Surgical treatment of post infarction ventricular aneurysm. Ann. Thorac. Surg., 21: 107-112, 1976.

24 MULLEN, D. C.; POSEY, L.; GABRIEL, R.; SINGH, H M.; FLEMA, R. J.; LEPLEY, D. - Prognostic considerations in the management of left ventricular aneurysms. Ann. Thorac. Surg., 23: 455-460, 1977.

25 NAJAFI, H.; MENG, R.; JAVID, H.; HUNTER, J. A.; GOLDIN, M.; MONSON, D. O.; NAJAFI, K. L. - Postmyocardial infarction left ventricular aneurysm. Cardiovasc. Clin., 12: 81-91, 1982
26 NICHOLOSI, A. C.; WENG, Z. C.; DETWILER, P. W. SPOTNITZ, H. M. - Simulated left ventricular aneurysm: an aneurysm repair in swine. J. Thorac. Cardiovasc. Surg., 100: 745-755, 1990.

27 NOVICK, R. J.; STEFANISZYN, H. J.; MORIN, J. E.; SYMES, J. R.; SNIDERMAN, A. D.; DOBELL, A. R. C. - Surgery for postinfarction left ventricular aneurysm: prognosis and long-term follow-up. Canad. J. Surg., 27: 161-167, 1984.

28 OKIES, J. E.; DIETL, C.; GARRISON, H. B.; STARR, A - Early and late results of resection of ventricular aneurysm. J. Thorac. Cardiovasc. Surg., 75: 255-260, 1978.

29 OLEARCHYK, A. S.; LEMOLE, G. M.; SPAGNA, P. M - Left ventricular aneurysm. Ten years experience in surgical treatment of 244 cases: improved clinical status, hemodynamics, and long-term longevity. $J$ Thorac. Cardiovasc. Surg., 88: 544-553, 1984.

RATCLIFFE, P. J.; KAY, P.; OLDERSHAWM P. J. - Longterm survival following left ventricular aneurysmectomy. J. Cardiovasc. Surg., 24: 461-466, 1983.

31 RIVERA, R. \& DELCAN, J. L. - Factors influencing better results in operation for postinfarction ventricular aneurysm. Ann. Thorac. Surg., 27: 445-450, 1979.

32 SCHLICHTER, J.; HELLERTEIN, H. K.; KATY, L. N. Aneurysm of the heart: a correlation study of 102 proved cases. Medicine, 33: 42-86, 1954.

33 SHATTENBERG, T. T.; GUILLANI, E. R.; CAMPION, B C.; DANIELSON, G. H. - Post-infarction ventricular aneurysm. Mayo Clin. Proc., 45: 13-19, 1970.

34 STEPHENSON, L. W.; HARGROVE, C. W.; RATCLIFFE M. B.; EDMUNDS Jr., L. H. - Srugery for left ventricular aneurysm: early survival with and without endocardial resection. Circulation, 79 (Supl. 1): 108-111, 1989

35 WALKER, W. E.; STONEY, W. S.; ALFORD, W. C.; BURRUS, G. R.; FRIST, R. A.; GLASSFORD, D. M.; THOMAS, C. S. - Techniques and results of ventricular aneurysmectomy with emphasis on anteroseptal repair. J. Thorac. Cardiovasc. Surg., 76: 824-831, 1978.

36 WRIGHT, J. S.; STACEY, R. B.; ALBRECHT, H.; MURTON, M. M. - Left ventricular aneurysm surgery: determinants of results. J. CardiovasC. Surg., 28: 85-88, 1987. 